\title{
Modeling ultrasonic attenuation coefficient and comparative study with the principal theoretical models of biphasic suspensions
}

\author{
A. Hamine ${ }^{1}$, B. Faiz ${ }^{1}$, A. Moudden ${ }^{1}$, G. Maze $^{2}$, O. Al gaoudi ${ }^{3}$. \\ I. Aboudaoud ${ }^{1}$, D. El abassi ${ }^{1}$ \\ ${ }^{1}$ Laboratoire de métrologie et de traitement de l'information(LMTI), faculté des sciences Agadir, Morocco. \\ ${ }^{2}$ Laboratoire d'ondes et milieux complexes (LOMC), université du Havre, France \\ ${ }^{3}$ Laboratoire de mécanique des procédés de l'énergie et de l'environnement (MP2E), école nationale des \\ sciences appliquées -Agadir-,Morocco.
}

\begin{abstract}
Many phenomena can be responsible for the attenuation of sound through the suspensions depending on the nature of the particles of the fluid and the frequency range of interest. In particular we can make a distinction between the diffusion mechanisms corresponding to a geometric redirection of the incident wave and the dissipative phenomena, like the thermal and viscous losses. In this work, we are interested in propagation of the ultrasonic waves into suspensions of clay rigid particles with a size between 1 and 50 microns, for which the thermal phenomena and visco-inertial dominate. In this case the dipole diffusion of the wave induced differential motion between the dispersed phase (clay grain) and the continuous phase (distilled water) is coupled to the viscous dissipation in the matching motion of this brake. In this paper, we present the main theories known in calculating the ultrasonic attenuation and velocity coefficient. Such theories permit to take accounts all the orders of interaction, unlike the theoretical of multiple diffusion that remains limited to lower concentrations. Finally, the results calculated by the principal theories will be compared against earlier experimental results obtained from this work.
\end{abstract}

Keywords: Attenuation measurements, attenuation coefficient, biphasic fluid, theoretical models, calculation of the attenuation and velocity coefficients

\section{INTRODUCTION}

Recently, there has been a great interest in the use of acoustics for the characterization of biphasic suspensions, by measuring the velocity and attenuation of ultrasonic waves. We can obtain the size and the concentration of the particles in suspension. The validity of this approach depends of the accuracy of experimental measurements of velocity and attenuation, made in the largest possible bandwidth. A number of theoretical models have been used in conjunction with experimental measurements to demonstrate the validity of these theories [1]. If the propagation of sound waves in dilute suspensions is relatively well modelized, the phenomena of interaction between the particles appearing in concentrated suspensions are the subject of many studies. Some authors use the theory of multiple diffusion to take into account the loop distribution [2-3]. Others are based on empirical models to incorporate viscous interactions [4-5]. In this paper, we present the main known theories in the study of biphasic suspensions. They allow taking account of all orders of interaction, unlike the theoretical of multiple diffusion remains limited to lower concentrations. The four models considered here are very variable in the complexity of the mathematical formulation, the assumptions made in each model and the physical phenomena that affect the acoustic propagation in two-phase media. The formulations and assumptions for each model are presented, followed by a comparison of the measurements results and calculated attenuation coefficient with theoretical models for three different suspensions.

\section{THE MAJOR THEORETICAL MODELS}

Six mechanisms considered in the fourths models are known in the interaction sound suspended matter:

- The viscous losses,

- the heat losses,

- scattering losses,

- intrinsic losses,

- electrokinetic losses,

- structural losses.

A sound wave interacts with the particles in suspension and in limited extent with the dispersing medium. The result of this interaction is a modification of the properties of the sound wave. A portion of the acoustic energy is unable to reach the transducer receiver. This part of the energy dissipated by conversion or 
diverted from its normal path between the transmitter and receiver by the diffusion, or any other geometric phenomenon. The viscous losses, thermal, intrinsic and diffusion are taken into consideration in the majority of acoustic analyzes, while structural and electrokinetic losses are either ignored or assumed to be negligible. However, structural losses can be of the utmost importance, especially for concentrated systems. The ultrasonic method is efficient to characterize dilute media.

The theory of sound propagation is well established for these biphasic systems where the particleparticle interaction is negligible. This theory was developed by Epstein and Carhart, and then generalized for suspensions and emulsions by Allegra and Hawley. This theory takes into account only the first four mechanisms mentioned above (viscous, thermal, intrinsic and diffraction), [6].

Viscous losses are due to the wave created by the oscillating particle in the acoustic pressure field. The wave amplitude decreases exponentially with distance from the surface of the particle. The characteristic size of this decrease is the viscous depth $\delta_{\mathrm{v}}$. It is given by the equation 1 :

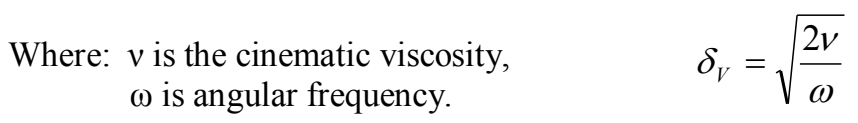

The thermal losses are caused to the temperature gradient close to the surface of the particle. These external and internal gradients are due to coupling between the thermodynamic pressure and temperature. Thermal depth is determined corresponding $\delta_{t}$, when the thermal wave enters the in the liquid, (2):

$$
\delta_{t}=\sqrt{\frac{2 \tau_{0}}{\omega \rho_{0} C_{p}^{0}}}
$$

Where: $\tau_{0}$ is the thermal conductivity, $C_{p}^{0}$ is the specific heat at constant pressure of the liquid, $\rho_{0}$ is the liquid density and $\omega$ the angular frequency.

Both of "viscous depth" and "thermal depth" parameters depend on the frequency. They decrease as the frequency increases. For specific frequencies, they become similar to the radius of the particle. The attenuation of the ultrasonic wave reaches its maximum at this frequency. The appropriate frequency is called "critical frequency". The ratio of the critical frequency depends to some extent at the ratio between the viscous and thermal depth. The viscous depth is larger than the thermal in the aqueous dispersions.

As a result, the critical frequency of thermal loss $W_{t h}$ is almost always lower than the critical frequency of viscous losses $W_{v}$. This significant difference between the critical frequencies implies that we can often neglect one of the phenomena in relation to the other. For example, viscous losses are dominant for dense, strong and rigid particles in a range of $1 \mathrm{MHz}$ to $30 \mathrm{MHz}$. The thermal losses are dominant for low-density dispersions and emulsions. The mechanism of diffusion loss is quite different from other mechanisms. It is similar to the light scattering. Acoustic diffusion does not produce acoustic energy dissipation. Particles simply redirect a portion of the acoustic energy so that part does not reach the receiving transducer. The work undertaken by Harker and Temple show that the theory developed for dilute systems was complete, while the concentrated systems, particularly polydisperse is far from being completed. They also showed that there are three different approaches which take into account the concentration [7]. The purpose of these theories is to incorporate the particle-particle interaction in the acoustic theory. A particle oscillating in acoustic pressure field in two-phase medium generates several waves, the waves of long distance compression, shear waves and short waves temperature. These waves acting on the adjacent particles can indeed, being considered as real particle-particle interactions. The particle-particle interaction through compression waves contributes to the mechanism of sound diffusion in a concentrate system. The treatment of multiple scattering is most suitable to describe this phenomenon. The effect of concentration on acoustics is negligible for colloids because the diffusion losses are manifested only for large particles and high frequencies. The particle-particle interaction across thermal waves and shear is more important for colloids. It affects the mechanisms of viscous and thermal losses predominate in the dispersions of fine particles. The particle-particle interaction creates a large shift of the acoustic spectrum to higher frequencies. The theory of sound propagation must take this phenomenon into account. Otherwise, the error in the calculation of the particle size can be huge. Fortunately, it appears that the sensitivity to thermal and viscous losses in the particle-particle interaction is different. The dilution experiments have shown that the mechanism of viscous loss is very sensitive to the hydrodynamic particle-particle interaction. At the same time, the contribution of the thermodynamic particle-particle interaction mechanism thermal loss is insignificant for volume fractions of $30 \%$ (by volume) [7].

\section{Model developed by Urick}

The most recent model and the easiest considered is that of Urick, which gives two separate equations, one of the ultrasonic velocity and the attenuation in a suspension, [8-9].The equation of velocity $V_{P}$, depends on 
the effective density and compressibility, when the effective properties of two component phases of suspension are additives. The propagation velocity was given by the equation 3 :

$$
V_{P}=\left(\rho_{\text {eff }} \beta_{\text {eff }}\right)^{-1 / 2}
$$

with $\rho_{e f f}=\rho(1-\phi)+\rho^{\prime} \phi$ and $\beta_{e f f}=\beta_{c}(1-\phi)+\beta_{c}^{\prime} \phi$

Where: $\rho_{\text {eff }}$ is the effective density, $\beta_{\text {eff }}$ is the effective compressibility,

$\phi$ is the solid volume fraction

$\rho^{\prime}$ and $\rho$ are the densities of the dispersed phase and the continuous phase,

$\beta_{c}^{\prime}$ and $\beta_{c}$ is the compressibility of the dispersed phase and continuous phase.

$$
\alpha_{S}=\frac{\phi}{2}\left[\frac{1}{3} k^{4} R^{3}+k(\sigma-1)^{2} \times \frac{36 b^{2} R^{2}(b R+1)}{[9(b R+1)]^{2}+\left[4 \sigma b^{2} R^{2}+2 b^{2} R^{2}+9 b R\right]^{2}}\right]
$$

With: $b=\left(\frac{\omega \rho}{2 \eta}\right)^{1 / 2}, \sigma=\frac{\rho}{\rho^{\prime}}$

Where: $\eta$ is the fluid viscosity,

$R$ is the radius of the particles.

The equation of the attenuation contains two terms, the first term is the energy loss by diffusion, it dominates for large particles at very high frequencies and also when the two phases have the same density. The second term describes both the energy lost due to the relative movement of the particles in the liquid phase. This attenuation is determined by the mass of the particles, the viscosity of the continuous phase and the ultrasonic frequency used.

$k_{s}=k^{2} \frac{\beta_{e f f}}{\beta_{c}}\left[1+\frac{3 \phi \zeta[b R(2 b R+3)+3 i(b R+1)]}{b R(4 \zeta b R+6 b R+9)+9 i(b R+1)}\right] \quad$ with $\quad \zeta=\frac{\rho^{\prime}-\rho}{\rho}$

\section{Model developed by Allegra ana nawley}

The work done by Allegra and Hawley has become a reference point in studies of ultrasound propagation in colloidal systems and biphasic [6]. That is due that the model is able to describe the effects of thermal loss and visco-inertial, under the assumption that the particles are spherical and monodisperse. Considering three equations of the mass conservation, the quantity of motion and energy. A complex equation of propagation of the velocity and the attenuation in the suspension is obtained. When an incident compression wave enters thought the solid-liquid interface of an isolated sphere suspended in a liquid, six waves are generated, compression waves, shear waves and thermal waves propagated on both sides of the solid-liquid interface in the suspension. The figure 1 shows schematically the direction of evolution of the different waves. The propagation equation is obtained by considering the relative amplitudes of six waves relative to the incident wave:

$$
\left(\frac{\beta}{k_{c}}\right)^{2}=1+\frac{3 \phi}{i k_{c}^{3} R^{3}} \sum_{n=0}^{\infty}(2 n+1) A_{n} \quad \text { with } \quad \beta=\frac{\omega}{V_{s}}+i \alpha_{s}
$$

Where: $k_{c}$ is the wave number of compression loss for a pure liquid.

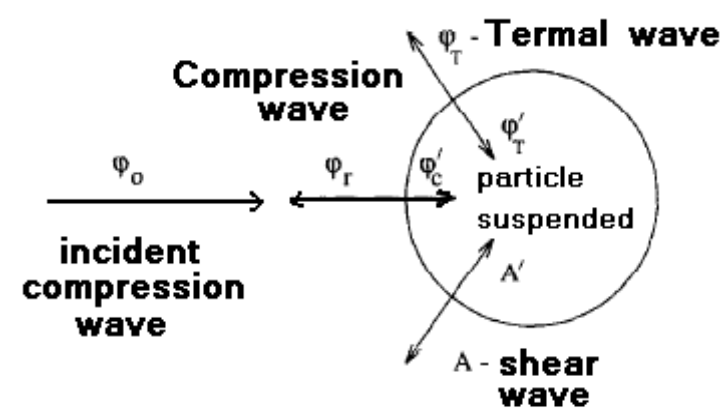

Fig. 1: The three types of waves dispersed inside and outside of an isolated particle, according by Allegra and Hawley [1]. 
The equation of propagation depends on the unknown coefficient $A_{n}$, resulting from the resolution of the equation in polar and spherical coordinates of the wave compression reflected in the interface liquid-solid. The An coefficient is obtained from the exact resolution of six boundary conditions that existed at the particle surface, which reduces the system of six equations with six unknown coefficients $A_{n}$ up $C_{n}^{\prime}$. These equations are based on the potential of different waves, spherical functions of Bessel and Hankel, densities and thermal, elastic properties of the two phases. These equations are given by the equation 7 to equation 10 :

$$
\begin{aligned}
&\left.b_{c}\left[j_{n}\left(a_{c}\right)+A_{n} h_{n}\left(a_{c}\right)\right]+b_{T} B_{n} h_{n}\left(a_{T}\right)=(-i \omega) \mid b_{n}^{\prime} A_{n}^{\prime} j_{n}\left(a_{c}^{\prime}\right)+b_{T}^{\prime} B_{n}^{\prime} j_{n}\left(a_{T}^{\prime}\right)\right] \\
& k\left\lfloor a_{c} b_{c}\left\lfloor j_{n}^{\prime}\left(a_{c}\right)+A_{n} h_{n}^{\prime}\left(a_{c}\right)\right\rfloor+\right.\left.B_{n} b_{T} a_{T} h_{n}^{\prime}\left(a_{T}\right)\right\rfloor=(-i \omega) k^{\prime}\left\lfloor A_{n}^{\prime} b_{c}^{\prime} a_{c}^{\prime} j_{n}^{\prime}\left(a_{c}^{\prime}\right)+B_{n}^{\prime} b_{T}^{\prime} a_{T}^{\prime} j_{n}^{\prime}\left(a_{T}^{\prime}\right)\right] \\
& a_{c} j_{n}^{\prime}\left(a_{c}\right)+A_{n} a_{c} h_{n}^{\prime}\left(a_{c}\right)+B_{n} a_{T} h_{n}^{\prime}\left(a_{T}\right)-C_{n} n(n+1) h_{n}\left(a_{s}\right) \\
&=(-i \omega)\left[A_{n}^{\prime} a_{n}^{\prime} j_{n}^{\prime}\left(a_{c}^{\prime}\right)+B_{n}^{\prime} a_{T}^{\prime} j_{n}^{\prime}\left(a_{T}^{\prime}\right)-C_{n}^{\prime} n(n+1) j_{n}\left(a_{s}^{\prime}\right)\right]
\end{aligned}
$$

$$
\begin{aligned}
& j_{n}\left(a_{n}\right)+A_{n} h_{n}\left(a_{c}\right)+B_{n} h_{n}\left(a_{T}\right)-C_{n}\left\lfloor h_{n}\left(a_{s}\right)+a_{s} h_{n}^{\prime}\left(a_{s}\right)\right\rfloor \\
& =(-i \omega)\left[A_{n}^{\prime} j_{n}\left(a_{c}^{\prime}\right)+B_{n}^{\prime} j_{n}\left(a_{T}^{\prime}\right)-C_{n}^{\prime}\left[j_{n}\left(a_{s}^{\prime}\right)+a_{s}^{\prime} j_{n}^{\prime}\left(a_{s}^{\prime}\right)\right]\right] \\
& \eta_{s}\left[\begin{array}{l}
{\left[\left(a_{c}^{2}-2 a_{c}^{2}\right) j_{n}\left(a_{c}\right)-2 a_{c}^{2} j_{n}^{\prime \prime}\left(a_{c}\right)\right]} \\
+A_{n}\left[\left(a_{s}^{2}-2 a_{c}^{2}\right) h_{n}\left(a_{c}\right)-2 a_{c}^{2} h_{n}^{\prime \prime}\left(a_{c}\right)\right] \\
+B_{n}\left[\left(a_{s}^{2}-2 a_{T}^{2}\right) h_{n}\left(a_{T}\right)-2 a_{T}^{2} h_{n}^{\prime \prime}\left(a_{T}\right)\right] \\
+C_{n} 2 n(n+1)\left[a_{s} h_{n}^{\prime}\left(a_{s}\right)-h_{n}\left(a_{s}\right)\right]
\end{array}\right] \\
& =A_{n}^{\prime}\left[\left(\omega^{2} \rho^{\prime} R^{2}-2 \mu^{\prime} a_{c}^{2}\right) j_{n}\left(a_{c}^{\prime}\right)-2 \mu^{\prime} a_{c}^{2} j_{n}^{\prime \prime}\left(a_{c}^{\prime}\right)\right] \\
& +B_{n}^{\prime}\left[\left(\omega^{2} \rho^{\prime} R^{2}-2 \mu a_{T}^{\prime 2}\right) j_{n}\left(a_{T}^{\prime}\right)-2 \mu^{\prime} a_{T}^{\prime 2} j_{n}^{\prime \prime}\left(a_{T}^{\prime}\right)\right] \\
& +C_{n}^{\prime} 2 \mu^{\prime} n(n+1)\left[a_{s}^{\prime} j_{n}^{\prime}\left(a_{s}^{\prime}\right)-j_{n}\left(a_{s}^{\prime}\right)\right] \\
& \eta_{s}\left[\begin{array}{l}
a_{c} j_{n}^{\prime}\left(a_{c}\right)-j_{n}\left(a_{c}\right)+A_{n}\left[a_{c} h_{n}^{\prime}\left(a_{c}\right)-h_{n}\left(a_{c}\right)\right] \\
+B_{n}\left[a_{T} h_{n}^{\prime}\left(a_{T}\right)-h_{n}\left(a_{T}\right)\right] \\
-\left(C_{n} / 2\right)\left[a_{s}^{2} h_{n}^{\prime \prime}\left(a_{s}\right)+\left(n^{2}+n-2\right) h_{n}\left(a_{s}\right)\right]
\end{array}\right] \\
& =\mu^{\prime}\left[\begin{array}{l}
A_{n}^{\prime}\left[a_{c}^{\prime} j_{n}^{\prime}\left(a_{c}^{\prime}\right)-j_{n}\left(a_{c}^{\prime}\right)\right] \\
+B_{n}^{\prime}\left[a_{T}^{\prime} j_{n}^{\prime}\left(a_{T}^{\prime}\right)-j_{n}\left(a_{T}^{\prime}\right)\right] \\
-\left(C_{n}^{\prime} / 2\right)\left[a_{s}^{\prime 2} j_{n}^{\prime \prime}\left(a_{s}^{\prime}\right)+\left(n^{2}+n-2\right) j_{n}\left(a_{s}^{\prime}\right)\right]
\end{array}\right]
\end{aligned}
$$

Allegra and Hawley have included the effects of heat transport using the first law of thermodynamics as a conservation equation of energy. They showed that the suspensions have almost similar densities for both phases, but very different thermal properties. And the attenuation and wave velocity dominated by contributions from the mechanisms of thermal loss. They also presented a good correlation with their model and results for suspensions of polystyrene particles.

\section{Model developed by Harker and Temple}

Harker and Temple considered that the acoustic propagation through suspensions from hydrodynamic perspective, [7]. They have led to equations representing the viscous force applied to the interface of each phase, the conservation of the quantity of motion for each individual phase. The simultaneous solution of these differential equations results in a complex propagation equation $k$ which is given by equation 11:

$$
k=\beta_{e f f} \omega^{2}\left[\frac{\rho\left[\rho^{\prime}+\left(\phi \rho^{\prime}+(1-\phi) \rho M(\omega)\right)\right]}{\left(\rho+(1-\phi) \rho^{\prime}\right)+\rho M(\omega)}\right]
$$

Where $M(\omega)$ is the coefficient of Couples force given by:

$$
M(\omega)=\frac{1+2 \phi}{2(1-\phi)^{2}}+\frac{1}{1-\phi} \frac{9 \varepsilon_{v}}{4}\left[1+\left(1+\frac{\varepsilon_{v}}{R}\right) i\right] \quad \varepsilon_{v} \text { the viscous depth. }
$$


The velocity and attenuation depend on the frequency. We find the expression of the frequency using the following formula:

$$
V_{P}(\omega)=\frac{\omega}{\operatorname{Re}[k]}, \quad \alpha_{s}=\operatorname{Im}[k]
$$

\section{THE CHANGES APPLIED TO MODELS}

The model developed by Allegra-Hawley and the Harker-Temple [6-7], can be modified to increase their applications to real systems. Harker and Temple included a term effective viscosity given by Equation 14:

$$
\eta_{\text {eff }}=\eta(1+K \phi)
$$

This term takes into account the effect of particles on the real viscosity of the liquid. It is a function of the volume fraction of the dispersed phase, the particle geometry and fluid viscosity. The form factor K, allows to model non-spherical particles. The Allegra and Hawley model can be reformulated for solid-solid dispersions. The diffusion coefficients An, have been incorporated into theories of multiple diffusion [11-12], by Holmes et al [13-14]. Both of theses models can be slightly modified to reflect the suspensions that have different particle size distributions.

\section{THE MODEL DEVELOPED TO CALCULATE THE ULTRASONIC ATTENUATION COEFFICIENT}

We have calculated the attenuation coefficient $\alpha$ using a simple theoretical model. The objective is to obtain an expression of the attenuation coefficient through a suspension of distilled water and clay grains. We can consider that the distilled water alone is an attenuating medium. If a small particle of clay prevents the wave reaching the receiving transducer, it will lose energy, and the loss by diffusion is the dominant process. In a suspension containing several particles, when a plane wave interacts with a particle, the wave is diffused in all directions, the diffusion is multiple.

We assume that:

- All the ultrasonic energy is lost to reach the receiver transducer.

- All particles have the same size.

- They all have the same mechanical properties.

The energy loss caused by $N$ particles can be written as follows, (15):

$$
d \xi_{\text {rétro }}=-N \xi_{\text {réfléchit }}
$$

With: $d \xi_{\text {rétro }}$ is the energy backscattered by each particle, $\xi_{\text {réfléchit }}$ is the reflected energy, and $N$ is the number of particles that are in the path of the wave.

To proceed with the calculation of the attenuation coefficient, first we must determine the number of particles in the path of the wave and the energy scattered by each particle. $\mathrm{Cm}$ is the mass fraction of particles in the suspension, given by equation 16 :

With: $\mathrm{m}_{\text {particle }}$ is the mass of particles in suspension,

$$
C_{m}=\frac{N m_{\text {particule }}}{m_{\text {liquide }}+N m_{\text {particule }}} \approx \frac{N m_{\text {particule }}}{m_{\text {liquide }}}
$$

$\mathrm{m}_{\text {liquide }}$ is the mass of the liquid phase in suspension.

The mass of the liquid is much bigger than the mass of the particle, for this reason that we have neglected the mass of the liquid. The figure 2 shows the schema assembly used in the calculation of the attenuation coefficient. 


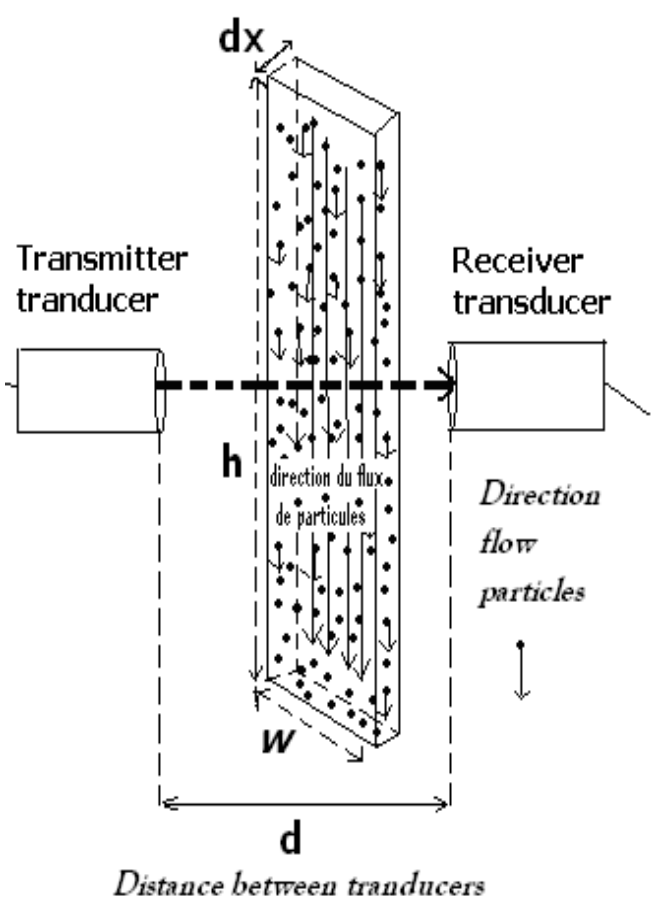

Fig. 2: Diagram assembly used in the calculation of the attenuation coefficient, (transmission method).

The dimensions of the measuring container used in the calculation of the attenuation coefficient $\alpha$ are: $\mathrm{w}=6 \mathrm{~cm}$ is the width of the container, $\mathrm{h}=10 \mathrm{~cm}$ is the height of the container, $\mathrm{dx}=1 \mathrm{~cm}$ thickness of the container, $d=15 \mathrm{~cm}$ is the distance between the transducers. The mass of the liquid inside the container is given by equation 17 :

$$
m_{\text {liquide }}=\rho_{\text {liquide }} V_{\text {liquide }}=\rho_{\text {liquide }} w \mathrm{~h} \mathrm{~d} x
$$

The mass of a particle is given by the equation 18 :

$$
m_{\text {particule }}=\rho_{\text {particule }} 4 / 3 \pi\langle a\rangle^{3}
$$

With : $\rho_{\text {liquide }}$ is the density of the liquid,

$\rho_{\text {particule }}$ is the density of the particle,

$<\mathrm{a}>$ is the mean radius of a particle.

We can estimate the number of $N$ particles by equation 19 :

$$
N \approx \frac{m_{\text {eau }}}{m_{\text {particule }}}=C_{m} \frac{3}{4} \frac{\rho_{\text {eau }} w \mathrm{~h}}{\rho_{\text {particule }} \pi\langle\mathrm{a}\rangle^{3}}
$$

After having estimated the number of particles in the mixture, we must determine how the clay particles change the path of the incident ultrasonic wave. We should also note that if the particles agglomerate, this will change the number of particles $N$.

When a plane wave of intensity I encounter incompressible rigid particles, the scattered intensity $I_{\mathrm{S}}$ is given by the equation 20, [18]:

$$
\frac{I_{s}}{I}=\frac{16 \pi^{4} f^{4}}{9 C^{4} r^{2}}\langle a\rangle^{6}(1-3 \cos \theta)^{2}
$$

With : $f$ is the frequency,

$C$ is the sound velocity in the medium,

$r, \theta$ are the radius and the angle in polar coordinates.

This expression is valid for the wavelength such only $(k a<<1)$,

Where: $k$ is the wave number

$a$ is the particle radius. 
Figure 2 shows the evolution of the backscattered intensity of a plane wave incident, [19].

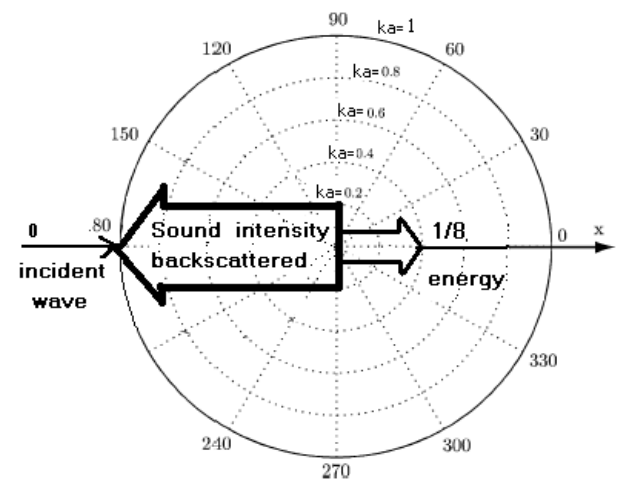

Fig.2: Incident plane wave from left to right in the figure, scattered by a particle, $(\mathrm{ka}=0.3)$.

Even if the equation 20 is given in polar coordinates $(r, \theta)$, it is valid in all three dimensions, due to the symmetry about the $\mathrm{x}$ axis of abscissas. As shown in figure 2, most of the energy is scattered and deflected in the opposite direction of the incident wave. In fact, only $1 / 8$ of the energy is diverted to the front (ie to the right of the figure). The backscattered intensity ratio is valid when the wavelength satisfies the following condition $(\mathrm{ka}<<1)$. The total backscattered energy is obtained by integrating the equation 8 on a half sphere with an origin in the same position as the particle in question, we have the equation 21 :

$$
\xi_{\text {rétrodiffisé }}=\int_{\pi / 2}^{\pi} I_{S}(r, \theta) 2 \pi r^{2} \sin \theta d \theta
$$

The intensity $I$ of the incident wave is related to the energy (22). When the frequency is replaced by the wavelength $\lambda=\mathrm{c} / \mathrm{f}$, then:

$$
I=\frac{\xi}{\pi\left(\frac{D}{2}\right)^{2}}=\frac{4 \xi}{\pi D^{2}}
$$

Where: $\xi$ is the incident plane wave at position $\mathrm{x}$,

$\mathrm{D}=16 \mathrm{~mm}$ is the diameter of the transmitting transducer.

The backscattered energy is now:

$$
\begin{gathered}
\xi_{\text {rétrodiffisé }}=\frac{16 \pi^{4} f^{4}}{9 C^{4}}\langle a\rangle^{6} I \int_{\pi / 2}^{\pi}(1-3 \cos \theta)^{2} 2 \pi \sin \theta d \theta \\
\xi_{\text {rétrodiffisé }}=\frac{114}{9} \quad \frac{\pi^{5}\langle a\rangle^{6}}{\lambda^{4}} I=\frac{152}{3} \frac{\pi^{4}}{\lambda^{4}} \frac{\langle a\rangle^{6}}{D^{2}} \xi
\end{gathered}
$$

Replacing equations (19) and (24) in equation (20), we obtain an ordinary differential equation of the energy loss the ultrasonic wave, (25).

$$
\begin{aligned}
& \frac{d \xi_{\text {ret }}}{\xi}=-\frac{152}{3} \frac{\pi^{4}}{\lambda^{4}} \frac{\langle a\rangle^{6}}{D^{2}} \times C_{m} \frac{3}{4} \frac{\rho_{\text {eau }} w h}{\rho_{\text {odbicule }} \pi\langle a\rangle^{3}} \\
& \frac{d \xi_{\text {rét }}}{\xi}=-A \frac{\langle a\rangle^{3}}{\lambda^{4}} \frac{\rho_{\text {eau }}}{\rho_{\text {oabicuó }}} C_{m} d x
\end{aligned}
$$

With : $A=38 \pi^{3} \frac{w h}{D^{2}}$, integration constant.

$$
\int_{\xi_{0}}^{\xi} \frac{d \bar{\xi}}{\bar{\xi}}=-\int_{0}^{x} A \frac{\langle a\rangle^{3}}{\lambda^{4}} \frac{\rho_{\text {eau }}}{\rho_{\text {particule }}} C_{m} d \bar{x}
$$

Where: $\bar{\xi}$ and $\bar{x}$ are the integration variables,

$\xi_{0}$ the transmitted energy at the position $\mathrm{x}=0$. 
After integration, we obtain the equation 28:

$$
\frac{\xi}{\xi_{0}}=e^{-C_{m} A \frac{\langle a\rangle^{3}}{\lambda^{4}} \frac{\rho_{e \alpha u}}{\rho}}=e^{-\alpha x}
$$

The attenuation in the suspension is given by equation 29 :

$$
\alpha=C_{m} A \frac{\langle a\rangle^{3}}{\lambda^{4}} \frac{\rho_{\text {eau }}}{\rho_{\text {patizule }}}
$$

We note that the calculated attenuation coefficient depends on the mass fraction of particles present in the suspension and the propagation distance of the wave between the transducers. For a measuring system witch the distance $d$ between the transducers and the container is constant (see Fig.1). The attenuation coefficient $\alpha$ is proportional only to the mass fraction $C_{m}$. The expression given by the equation (29), is valid when the mass fraction of the particles is low, for assuming that there is no multiple scattering. In addition, this model developed in this work, we assumed that there is a constant mean particle radius and the length of ultrasonic wave is constant.

\section{RESULTS AND DISCUSSION}

\section{Comparison of the measured attenuation coefficient and the calculated coefficient}

We compared the experimental attenuation with the theoretical calculated coefficient, we have remark that the attenuation coefficient $\alpha$ varies linearly with the concentrations of clay grains, (Fig. 3). We also found that there is a perfect resemblance between the calculated theoretical model and the experimental attenuation coefficient, only for dilute suspensions of clay.

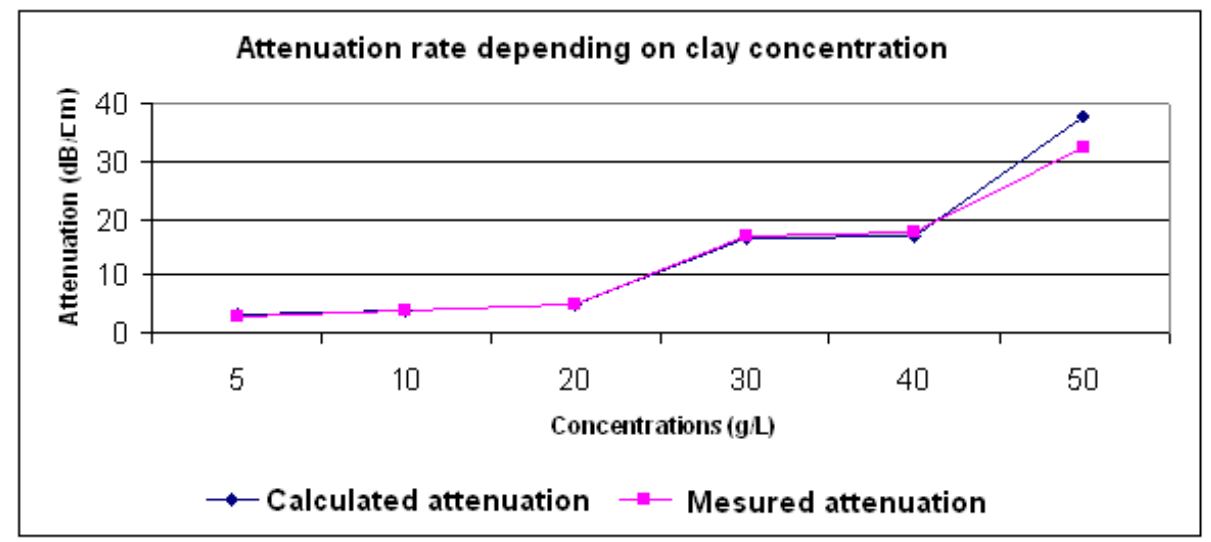

Fig. 3: The attenuation based on the clay concentration.

\section{Comparison of principal theoretical models and measured results for three suspensions}

In the following, we present a comparative study between the four principal models and the measurement results obtained for three suspensions of different concentrations $(5 \mathrm{~g} / \mathrm{l}, 40 \mathrm{~g} / \mathrm{l}, 50 \mathrm{~g} / \mathrm{l})$. Theses suspensions have a very different density.

\subsection{Comparison of the velocity and attenuation for a suspension of $5 \mathrm{~g} / \mathrm{l}$ with four models}

We present in this paragraph, the comparison between the values of the models presented above, and the measured values of the velocity and attenuation for a concentration of $5 \mathrm{~g} / \mathrm{l}$. From the analysis of the curves of the figure 4 and 5 , we remark that the models coincide with the measured values either speed or attenuation. The values of the velocity increase as the frequency increases and vary linearly. In the other hand, we remark that the curve 5 shows a constant attenuation in two distinct interval, the first one is situated between 2 to $4 \mathrm{MHz}$ and the second from 8 to $12 \mathrm{MHz}$, but the values between 4 and $8 \mathrm{MHz}$, the attenuation increases until it reaches the constant $11.5 \mathrm{~dB} / \mathrm{cm}$. 


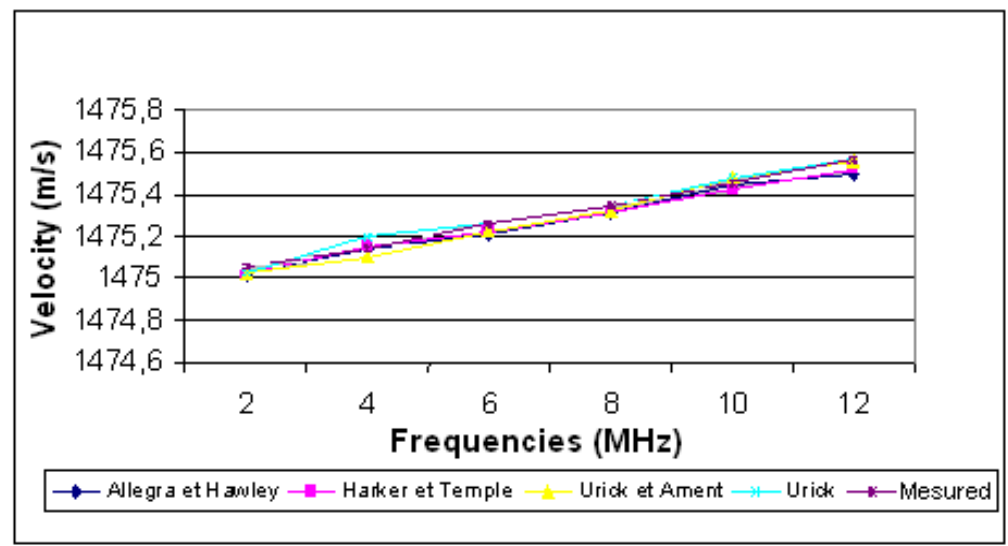

Fig. 4: The evolution of sound velocity as a function of the frequency of the four models and measured speed from the experience (a suspension of $5 \mathrm{~g} / \mathrm{l}$ ).

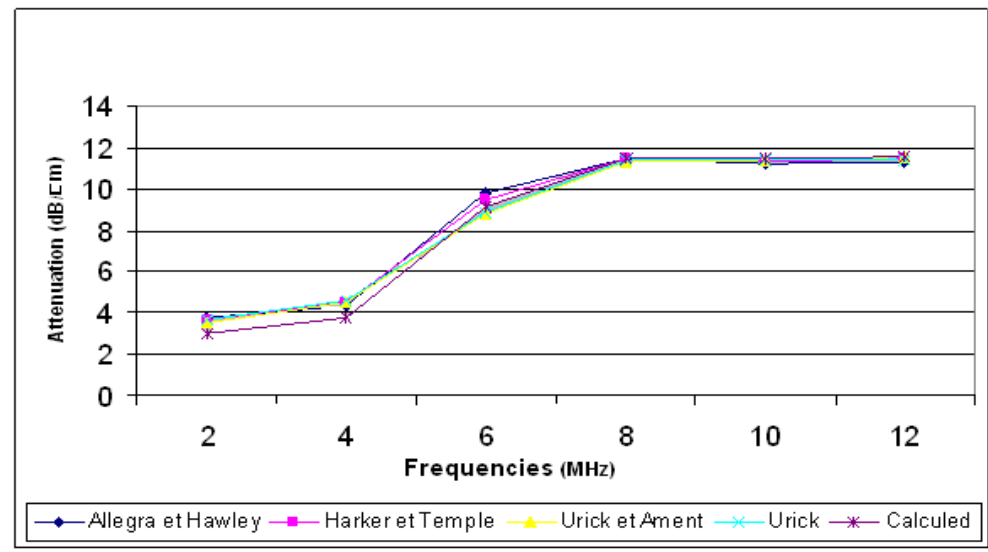

Fig. 5: The evolution of the attenuation as a function of the frequency of the four models and measured attenuation from the experience (a suspension of $5 \mathrm{~g} / \mathrm{l}$ ).

\subsection{Comparison of the velocity and attenuation for a suspension of $40 \mathrm{~g} / \mathrm{l}$ with four models}

The figures 6 and 7 represent the phase velocity and the attenuation of the principal models and the measured values from experience versus frequencies. The analysis of these curves shows that all the models follow perfectly the measured values of velocity and attenuation. The analysis of the curve of the figure 6 shows that the value of the speed varies linearly with frequencies and increase progressively as the frequency of the transducer increases. For against, the curve of the figure 7 shows that the attenuation increases in steps depending on the frequencies, the first step is situated between 2 and $4 \mathrm{MHz}$ and the second between 6 and 8 $\mathrm{MHz}$, but the frequencies between 4 and $8 \mathrm{MHz}$ the attenuation increases rapidly.

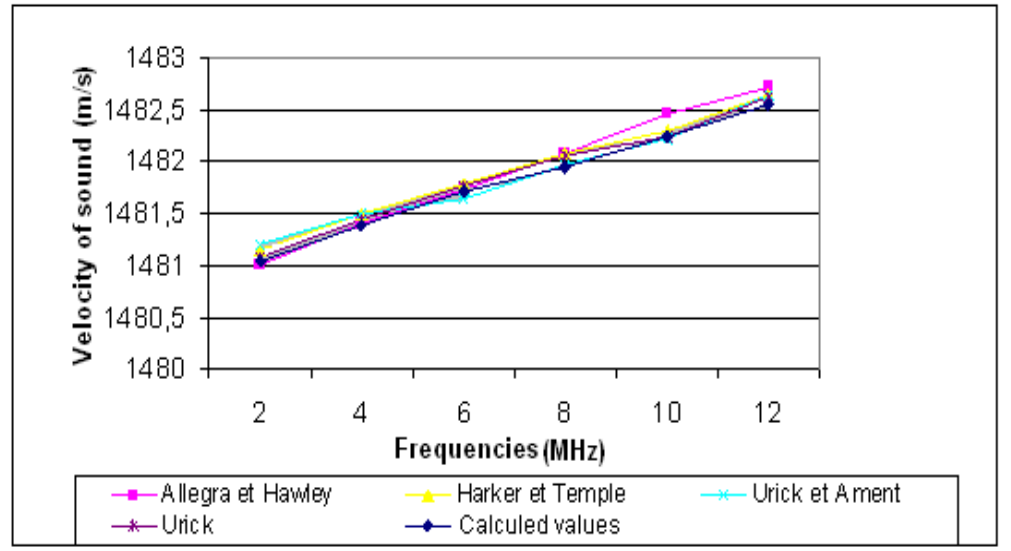

Fig. 6: The evolution of velocity as a function of frequency for the models and the experience for a suspension of $40 \mathrm{~g} / \mathrm{l}$. 


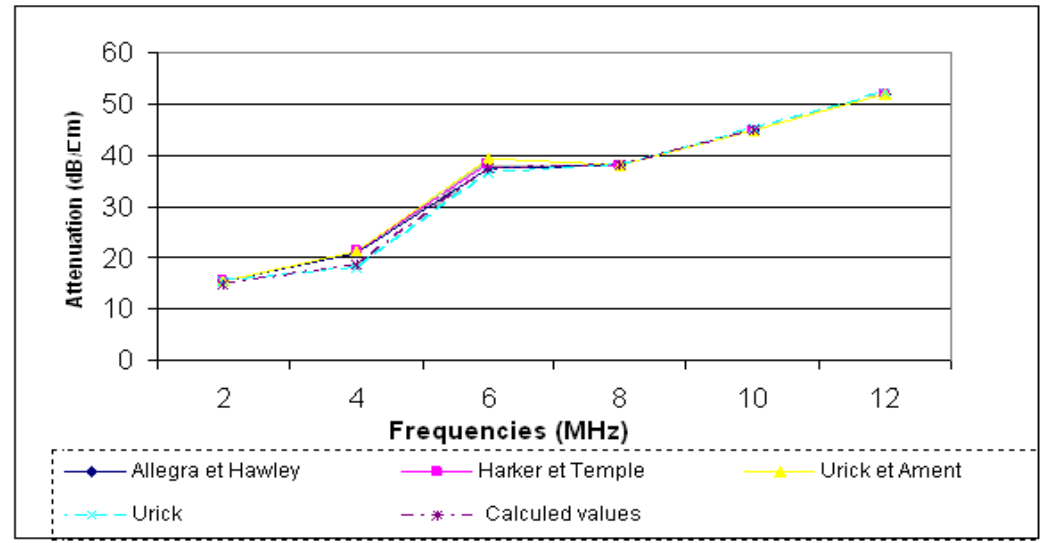

Fig. 7: The evolution of attenuation versus frequency of models and measured values for a suspension of $40 \mathrm{~g} / \mathrm{l}$.

The figures $(8,9)$ represent the values of the phase velocity and attenuation respectively, of the models and measured values from experience. Based on the analysis of the curve of the figures (Fig. 8) and (Fig.9), we note that the model move away and deviates from the measured values of phase velocity and attenuation. We can conclude that when the clay concentration increases in suspension, the values of experience do not follow any of the models. This is explained by the fact that when it exceeds the amount of $50 \mathrm{~g} / \mathrm{l}$ considered as level of the concentrate suspension of clay, we must use other models that take into account. In such suspension several phenomena must be taken into consideration.

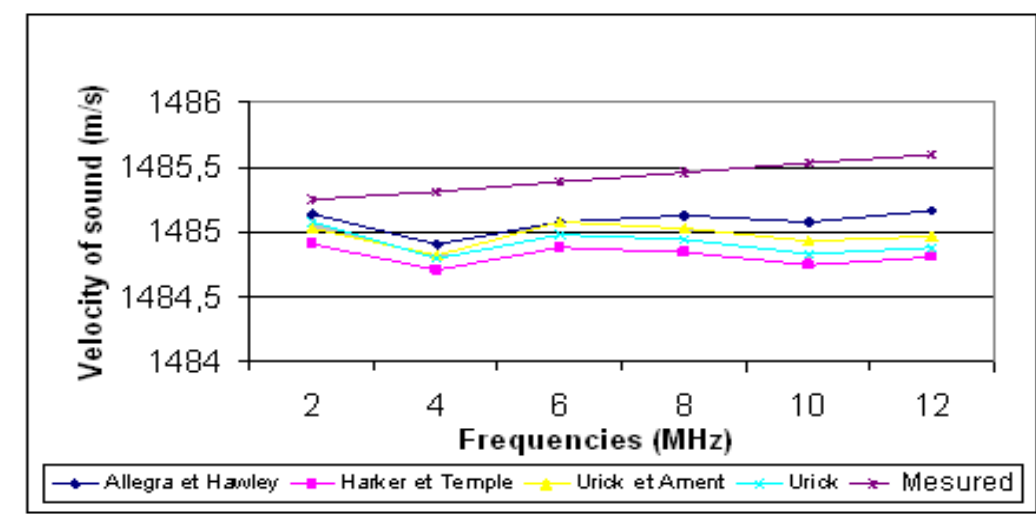

Fig. 8: The evolution of velocity as a function of frequency for the models and the experience for a suspension of $50 \mathrm{~g} / 1$.

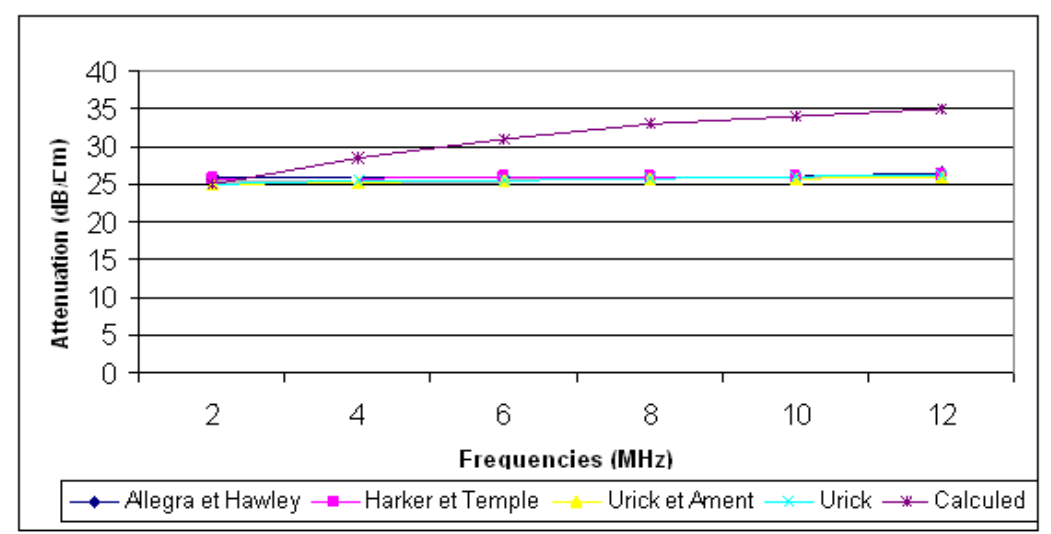

Fig. 9: The evolution of attenuation versus frequency of models and measured values for a suspension of $50 \mathrm{~g} / \mathrm{l}$.

\section{Comparison of calculated and measured attenuation values with four models}

From the experiments made in our laboratory we found that the attenuation is a more reliable and accurate than the phase velocity to control the sedimentation of clay grains parameter. This can be explained by 
that the given values of the phase velocity of the four models deviate them present unstable speed variations value around $1485 \mathrm{~m} / \mathrm{s}$.

In table 1, we show a comparison of the values of attenuation calculated and measured with the principal models. The figure 10 shows a similar linear attenuation behavior for all the concentrations, but from the concentration of $40 \mathrm{~g} / \mathrm{l}$, considered like a limit of a concentrate suspension, we found that:

- The values of the measured and calculated attenuation deviate them about $5 \mathrm{~dB} / \mathrm{cm}$,

- the measured values deviate from four models about $10 \mathrm{~dB} / \mathrm{cm}$,

- the calculated values differ from the models approximately by $15 \mathrm{~dB} / \mathrm{cm}$.

Tab. 10: The values of the calculated attenuation measured and those models.

\begin{tabular}{|c|c|c|c|c|c|c|}
\hline $\begin{array}{c}\text { Concentration } \\
(\mathbf{g} / \mathbf{l})\end{array}$ & $\begin{array}{c}\text { Calculed } \\
\text { Attenuation }\end{array}$ & $\begin{array}{c}\text { Mesured } \\
\text { attenuation }\end{array}$ & Allegra \& Hawley & $\begin{array}{c}\text { Harker \& } \\
\text { Temple }\end{array}$ & $\begin{array}{c}\text { Urick \& } \\
\text { Ament }\end{array}$ & \begin{tabular}{c} 
Urick \\
\hline 5
\end{tabular} \\
\hline 3,5 & 3,8 & 7,1 & 7,159 & 7,2 & 7,256 \\
\hline 40 & 17 & 17,5 & 19 & 19,3 & 18,3 & 18,3 \\
\hline 50 & 38 & 32,5 & 25 & 25,3 & 25,24 & 25 \\
\hline
\end{tabular}

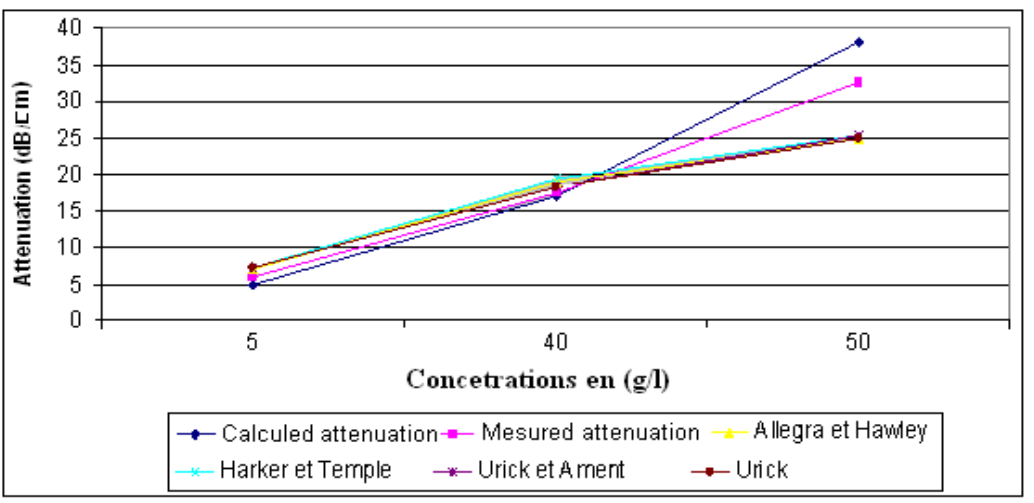

Fig. 10: The evolution of the calculated attenuation, measured attenuation and attenuation of the models according to the different concentrations

The experimental comparisons made between the different models have shown that the phase velocity and attenuation are similar except for the case of the suspension of $50 \mathrm{~g} / \mathrm{l}$.

In this case, where the concentration of clay is high, the values of the attenuation of the models are different from the values measured and calculated. This is due to the thermal effects of the two phases, and it become more important for concentrated suspensions of clay.

Under these conditions, the mechanisms of thermal losses considered in the four models and especially the Allegra and Hawley model, gave a dominant contribution to the velocity and the attenuation. For concentrated suspensions of clay, it was a big difference between the constituent of the suspension phases in thermal properties and densities. The first three models show a similar behavior intended to screw the attenuation. Contrariwise, the calculated and measured values differ with a significant scale from the other models. It indicates the importance of including process thermal losses in the calculations.

\section{CONCLUSION}

In this work, first we presented the studies of the major theory in acoustic wave propagation in suspensions of spherical particles. In the second, we have described the diffusion theory of a plane wave from a single particle, which allows to calculate the expression of the coefficient of attenuation of the wave through a suspension of clay particles.

In the third, we have compared the principal models that describe the propagation of ultrasound in biphasic media. The models differ in the complexity of their mathematical formulations. The application of one of these models to real suspensions is limited by the implicit assumptions in the calculations. The simulated comparison of the phase velocity and attenuation of the ultrasound is presented for three suspensions which are physically diverse. The experimental comparisons between the different models have shown that the phase velocity and attenuation are similar except for the case of the suspension of $50 \mathrm{~g} / \mathrm{l}$. In that case the clay concentration is high, the measured values of the phase velocity and attenuation are different from values obtained using four models one hand and on calculated values on the other hand. This was especially due to thermal effects and visco-inertial effects. They become significant between the two phases for concentrated suspensions and also show a big difference between the two phases of perspective thermal properties and densities. The first three models show a similar behavior for the phase velocity and attenuation. For against the measured and calculated values diverge from these models. 
Under these conditions, the thermal losses mechanisms considered give a dominant contribution to the phase velocity and attenuation. This indicates the importance of including thermal losses process in the calculations.

Allegra and Hawley model gives a full and adequate interpretation of the acoustic propagation through the less concentrated clay suspensions. Regarding concentrated suspensions of clay, we must appeal to other models.

\section{REFERENCES}

[1] M J.R. Allegra and S.A. Hawley, J. Acoust. Sot. Am. 51, (1972) 1545.Note that the journal title, volume number and issue number are set in italics.

[2] H. Lamb. Hydrodynamics. Dover Publications, New York, 1945.

[3] L.L. Foldy . The multiple scattering of waves. I. General theory of isotropic scattering by randomly distributed scatterers. Phys. Rev., $67: 107-119,1945$. M. Lax. Multiple scattering of waves. II. The effective field in dense systems. Phys. Rev.,85 :621-629, 1952 .

[4] A.S. Dukhin and P .J. Goetz.Acoustic spectroscopy for concentrated polydisperse colloids with high density contrast. Langmuir, 12 :4987-4997, 1996.

[5] D.J. McClements, Y . Hemar, and N. Herrmann. Incorporation of thermal overlap effects into multiple scattering theory . J. Acoust. Soc. Am., 2 :915-918, 1999.

[6] A.H. Harker and J.A.G. Temple, J. Phys. D: Appl. Phys. 21

[7] R.J. Urick, J. Appl. Phys, 18 (1947) 983.

[8] R.J. Urick, J. Acoust. Sot. Am. 20 (1948) 283.

[9] H. Lamb, Hydrodynamics, 6th edition (Dover Publications, New York, 1945, sections (361-363).

[10] A.K. Holmes, R.E. Challis and D.J. Wedlock, J. Colt. Int. Sci 156 (1993) 261.

[11] J.C. Austin, A.K. Holmes, J.S. Tebbutt and R.E. Challis, Ultrasonics 34 (1996) 369 (this issue).

[12] P. Lloyd and M.V. Berry, Proc. Phys. Sot. 91 (1967) 678.

[13] Dukhin, A. S., Shilov, V. N. \& Borkovskaya Yu. "Dynamic Electrophoretic Mobility in Concentrated Dispersed Systems. Cell Model.", Langmuir, 15, 10, 3452-3457, 1999.

[14] J. C. Austin, A. K. Holmes, J. S. Tebbutt, « Ultrasonic wave propagation in colloid suspensions and emulsions: recent experimental results »,Ultrasonics, Volume 34, Issues 2-5, June 1996, Pages 369-374.

[15] Savéry, D. and Cloutier, G., A point process approach to assess the frequency dependence of ultrasound backscattering by aggregating red blood cells, Journal of the Acoustical Society of America, 110 (2001), pp. 3252-3262.

[16] A. hamine and al, "Ultrasonic Attenuation Measurement Method for Characterization of Clay Particles Mass Fraction in Dilute Suspension"; Journal High Temperature Materials and Processes". Volume 28, Issue 6, Pages 369-378, March 2011.

[17] A. hamine and al, "Ultrasonic Technique for the Quality Control of Water Containing Clay"; J. Acoust. Soc. Am. Volume 123, Issue 5, pp. 3233-3233 (2008).

[18] D.J. McClements, P. Fairley, “Ultrasonic Pulse Echo Reflectometer”, Ultrasonics, 29, 58-60, 1991.

[19] Twersky, V., Low-frequency scattering by correlated distributions of randomly oriented particles, Journal of the Acoustical Society of America, 81 (1987), pp. 1609-1618. 